\title{
Current Status of Distal Embolization in Femoropopliteal Endovascular Interventions
}

Vascular and Endovascular Surgery

2018, Vol. 52(6) 440-447

(C) The Author(s) 2018

Reprints and permission:

sagepub.com/journalsPermissions.nav DOI: I0.I I77/I5385744|8764050

journals.sagepub.com/home/ves

(S)SAGE

\author{
Alessandro Cannavale, MD, EBIR', Mariangela Santoni, MD², \\ Marianna Gazzetti, MD, $\mathbf{P h D}^{3}$, Carlo Catalano, $\mathbf{M D}^{2}$, \\ and Fabrizio Fanelli, MD, EBIR ${ }^{4}$
}

\begin{abstract}
The application of advanced endovascular techniques in very complex femoropopliteal atherosclerotic lesions has shown to expose patients to a higher risk of distal embolization (DE). This complication can affect both the short- and long-term outcomes, leading to worsening ischemia, early minor/major amputation, and longer hospital stay. Recently, there has been an increasing body of evidence on pathophysiology and clinical-radiological management of DE that however has not been systematically addressed by guidelines.

The aim of this review was to analyze the current evidence outlining definition and classification, risk assessment, prevention, and management strategies of DE in femoropopliteal endovascular interventions.
\end{abstract}

\section{Keywords}

distal embolization, angiography, complication, thromboembolism, computed tomography angiography

\section{Introduction}

Due to the advancement in instrument technology and operators skills, endovascular therapies are now considered as the first-line approach even in complex femoropopliteal arterial steno/occlusive disease. ${ }^{1}$ According to this, distal embolization (DE) has shown an increasing incidence, ranging from $50 \%$ to $100 \%$, as detected by embolic filter protection or Doppler studies. $^{2-5}$ However, guidelines reported frequency of clinically significant DE between $1.6 \%$ and $2.4 \%$ of cases and up to $24 \%$ in thrombolysis for acute limb ischaemia. ${ }^{2,6,7}$

Patients experiencing DE will have longer hospital stay and a higher risk of worsening ischemia and limb loss. ${ }^{7}$ Major amputation rate may vary from $5 \%$ to $30 \%$ over 24 months. ${ }^{7,8}$ For these reasons, rigorous planning of endovascular/surgical approach with careful review of clinical history and imaging findings should be advocated. ${ }^{3}$ Over the last decade, there has been an increasing evidence on the pathophysiology and radiological-clinical management of DE; however, this information has not been gathered into a clear consensus on definition, predisposing factors, prevention techniques, and bailout treatment.

The aim of this review was to overview the current evidence and outline the latest evolvements on the prevention and treatment of DE.

\section{Definition and Classification}

The DE or thromboembolism is defined as the dislodgement of atherosclerotic material (plaque debris and/or thrombus) to the distal vessels caused by the manipulation of endovascular devices. $^{2}$

It is mostly made of an aggregate of plaque components (fibrin, calcified deposits, cholesterol clefts, and inflammatory and endothelial cells) and thrombus that may form macro- or micro-emboli of $>100$ and $<100 \mu \mathrm{m}$, respectively. ${ }^{5}$

DE can be classified as flow-limiting and non-flow-limiting.

The flow-limiting DE (or clinically significant DE) is characterized by a certain amount of debris causing clinically significant acute ischemia to the lower $\operatorname{limb}$ /foot, which requires prompt intervention to prevent further sequelae, as worsening ischemia or limb loss. This can be easily recognized during the angiogram as none or limited flow is observed in one or more below knee vessels. ${ }^{8-12}$ In a large registry, ${ }^{7}$ clinically significant DE occurred in up to $68 \%$ of all procedures with DE (DE

\footnotetext{
'Department of Radiology, Interventional Radiology Unit, NHS Greater Glasgow and Clyde, Glasgow, United Kingdom

${ }^{2}$ Department of Radiological Sciences, "Sapienza" University of Rome, Rome, Italy

${ }^{3}$ Vascular Surgery Services, Villa Stuart Medical Hospital, Rome, Italy

${ }^{4}$ Department of Interventional Radiology, Azienda Ospedaliera Universitaria "Careggi", Florence, Italy
}

\section{Corresponding Author:}

Alessandro Cannavale, Department of Radiology, Interventional Radiology Unit, NHS Greater Glasgow and Clyde, Queen Elizabeth University Hospital, I345 Govan Rd, Govan, Glasgow G5I 4TF, United Kingdom.

Email: alessandro.cannavale@hotmail.com 
occurred in 188/10.875 procedures) and required treatment with either endovascular (57\%) or surgical $(11 \%)$ approach.

The non-flow-limiting DE does not cause limitation of flow, usually not requiring immediate intervention during the procedure; hence, it has minor clinical relevance. Non-flow-limiting debris dissolves over time, without any consequences in the long-term follow-up in terms of primary patency, assisted primary patency, or secondary patency. ${ }^{8,9}$

During an angiogram, non-flow-limiting DE is shown as the presence of some debris in the infragenicular vessels (filling defects), but not causing significant stenosis or flow impairment.

\section{Risk Assessment}

In elective femoropopliteal endovascular procedures, identifying predicting factors of DE is paramount to identify patients at higher risk of DE. The most crucial predicting factors are related to the different types of lesions and devices used.

In fact, high-risk procedures may benefit from a different clinical approach, choice of devices, eventual joined surgical/ endovascular approach, and the use of prophylactic systems.

\section{Types of Atherosclerotic lesion}

Clinical presentation. Atherosclerotic lesions may be defined according to the time of clinical presentation as (a) hyperacute ( $<24$ hours), (b) acute A (1-7 days), (c) acute B (8-14 days), (d) subacute (14 days-3 months), and (e) chronic ( $>3$ months). ${ }^{13}$ This classification is particularly useful, given the reported better efficacy of thrombolysis in the treatment of acute limb ischemia, compared to older lesions. ${ }^{13,14}$

There is also an additional clinical presentation due to the onset of acute/subacute symptoms on an underlying chronic disease.

However, the description of symptoms as made by patients may often be not precise, so that a clear timing of the lesion cannot be defined only according to the clinical history. In example, this may happen when it is required to differentiate between the subacute and chronic presentation or to identify the acute/subacute on chronic symptomatic disease.

Imaging characteristics. Computed tomography (CT) or magnetic resonance imaging appearances of these different lesions may offer additional judgment criteria in view of the surgical/endovascular treatment.

The acute atherosclerotic disease may have an embolic or thrombotic presentation.

In the embolic lesion, there is a centered rounded filling defect with "ring" perfusion in axial view. ${ }^{14,15}$ Otherwise, in the acute thrombotic disease, there is complete obliteration of the vessel lumen and it may often be on a preexisting chronic disease or even more commonly in stents, stent grafts, or bypass grafts. Usually, none or few collaterals are present on CT angiogram. ${ }^{15}$
The subacute lesions are characterized mainly by thrombosis, associated with expansion of the vessel size when compared to the normal counterpart and there are some collaterals (Figure 1). Also, there could be an overt or subtle enhancement of the vessel wall, better visible on the axial view (due to congestion of the vasa vasorum). The risk of DE in this type of lesion is higher than in the classic chronic occlusion ${ }^{11,16}$ and the endovascular treatment by classic means is strongly discouraged because of the high risk of DE. ${ }^{16}$ Another suggestive sign of acute/subacute occlusion is the easy passage of the wire during angiography, which is commonly called the "traversal wire test."17

Several authors recognize that the acute and the subacute lesions are those with a higher risk of flow-limiting DE, reporting an incidence ranging from $2.3 \%$ to $9.8 \%$. $^{8,9,11}$ Calcifications may sometimes be present in the context of acute/subacute disease, but this is more often the case of the acute/subacute event superimposed on a condition of chronic atherosclerotic disease, such as stenosis, multiple stenoses, or occlusion. In this case, the aspect on CT angiography is a mixed lesion that mimics the chronic occlusion but may contain recent thrombus material. There are no specific studies on this particular type of lesion: It has similar risk of DE to the pure acute and subacute lesions, but it might be more challenging to identify.

Chronic disease is classically represented by a different amount of calcified and soft atherosclerotic plaques. There are several and large collaterals due to the long-standing occlusion/ stenosis (Figure 2). ${ }^{15}$

Chronic lesions were found to cause DE to a lesser degree than acute or subacute stenosis. Nevertheless, DE may occur especially in long (>100 mm) stenosis and occlusions (TASCD lesions; Figure 2). ${ }^{5}$ In fact, chronic total occlusions (CTO) are composed mainly by hard debris but may contain a certain degree of thrombus, especially in case of occluded stents and stent grafts. ${ }^{17}$ In fact, Zeller et al ${ }^{18}$ showed the presence of acute or chronic thrombus in chronic lesions, consisting of larger particles captured by the balloon based filter. Accordingly, Shammas et al found that longer lesions (130.0 \pm $123.3 \mathrm{~mm}$ ) reduced pretreatment peripheral Thrombolysis In Myocardial Infarction (TIMI) score (this score indicates the flow before treatment of DE, as normal/brisk: TIMI 3, slow, but fills the vessel completely: TIMI 2; and sluggish, partial filling or no flow: TIMI 1 and 0), more evidence of angiographic thrombus, and less frequent history of chronic symptoms are factors predicting higher risk of flow-limiting DE. ${ }^{11}$ Lesion characteristics at high risk of DE are resumed in Table 1.

\section{Types of Device}

With regard to the devices, Lam et al identified stent deployment, along with atherectomy and excimer laser devices as the major factors causing DE, compared to wire crossing and angioplasty. ${ }^{4}$ In particular, stent deployment showed 30.9 embolic signals, which was significantly more than angioplasty and wire passage. 


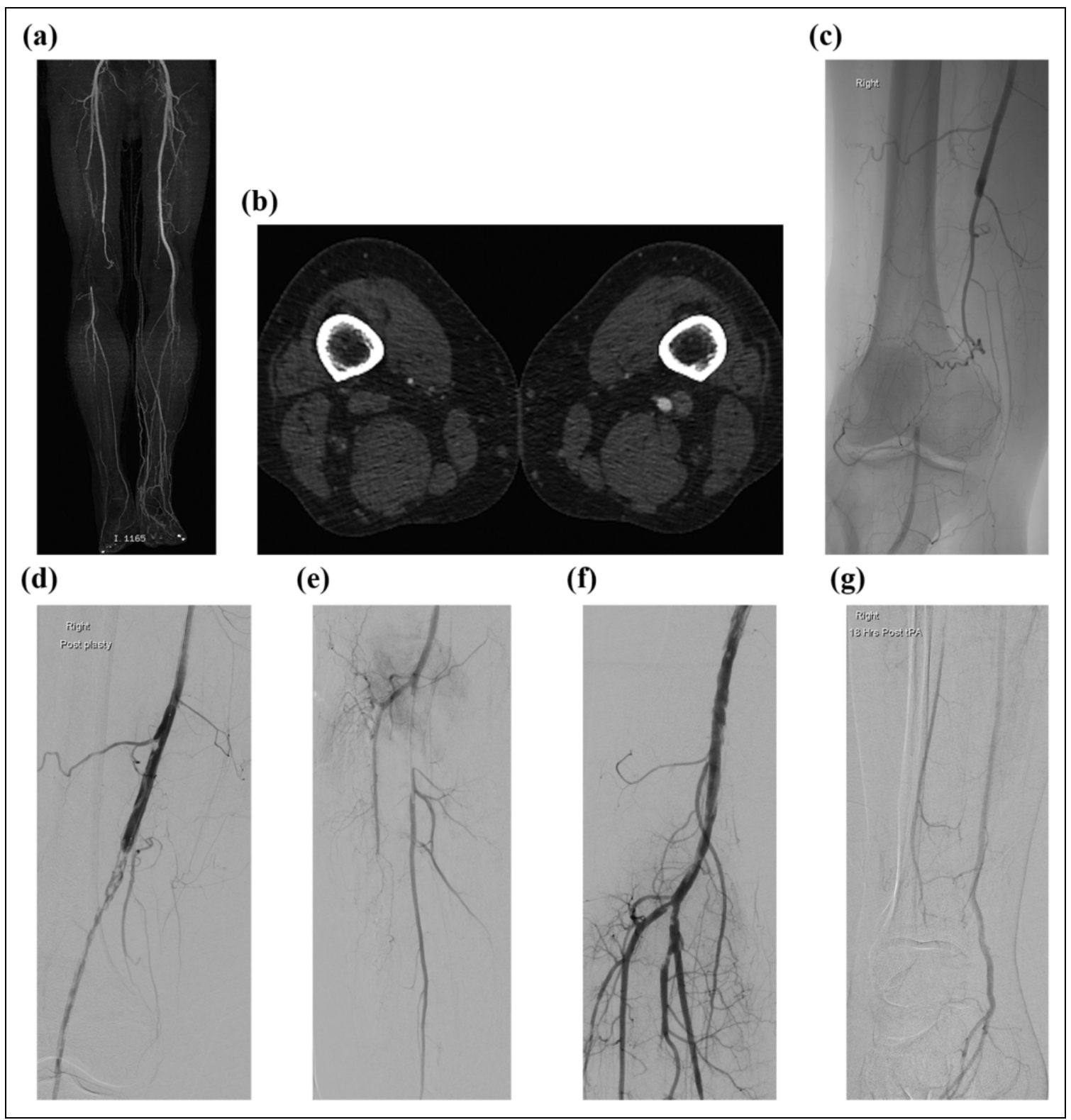

Figure I. A 50-year-old male with strong smoking habit, referring worsening right leg claudication and new onset of rest pain in the foot in the last 4 months. a, CT angiogram MIP reconstruction shows occlusion in the right SFA adductor canal involving the proximal popliteal artery. Neither many collaterals nor calcifications are observed. There is a two-vessel runoff via the posterior tibial and peroneal artery. $b$, On the axial image, the occluded artery seems to be slightly expanded as compared with the left SFA and has subtle wall enhancement. c, The antegrade angiogram confirms the long distal SFA occlusion that is easily crossed with the wire and balloon angioplasty at $7 \mathrm{~mm}$ is performed. Dislodgment of thrombus is noted along the SFA (d), then distally causing acute occlusion of the posterior tibial artery and peroneal artery (flow-limiting DE, e). e, Prompt infusion of $5 \mathrm{mg}$ of rTPA was performed through a straight catheter with side holes that is then left positioned in the peroneal artery also infusing in the posterior tibial artery for a total of 18 hours at I mg/h rate of rTPA. $\mathrm{f}$, g, Final angiograms shows satisfactory recovery of the below knee vessels. CT indicates computed tomography; SFA, superficial femoral artery; MIP, maximum intensity projection; DE, distal embolization; rTPA, recombinant tissue plasminogen activator.

Generally, atherectomy devices have been linked with a high incidence of significant DE, up to $56 \% .^{19,20,28}$ Large registries and recent trials reported similar incidence of DE in different types of atherectomy devices as excisional (ie, SilverHawk, Covidien/Medtronic, Minneapolis, MN, USA), orbital (ie, DiamondBlack 360, Cardiovascular Systems, Inc, St Paul, Minnesota, USA), and laser and Jetstream (Boston Scientific, Marlborough, Massachusetts, USA) atherectomy devices, ranging from $2.6 \%$ to $5.1 \%{ }^{7,29-31}$
An incidence of $8.3 \%$ has been reported for laser atherectomy in in-stent restenosis. ${ }^{19}$ Only Shrikhande et al ${ }^{9}$ in a prospective registry reported a significantly higher incidence of DE in Jetstream and Diamond Black (orbital; 22\% and 22\%, respectively) than Laser and Silverhawk atherectomy (3.6\% and $1.9 \%$, respectively).

Most studies assessing the effectiveness of atherectomy devices strongly recommend the use of embolic protection 
(a)

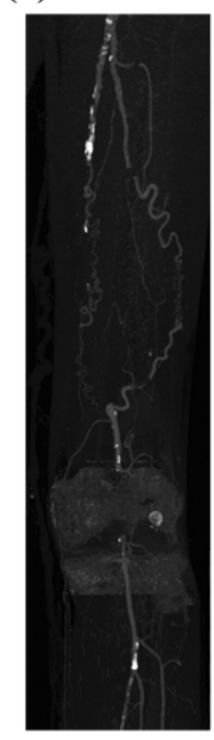

(c)

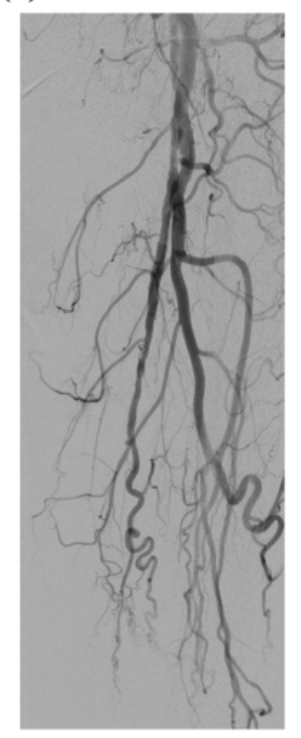

(d)

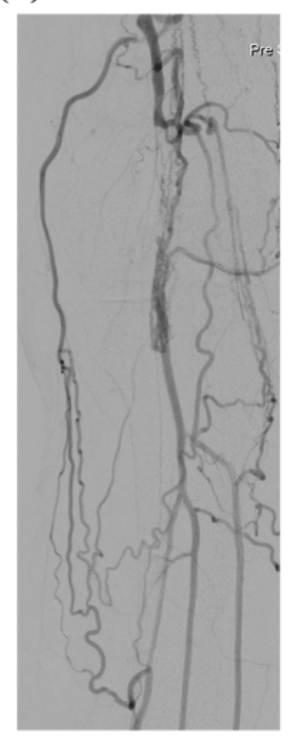

(e)

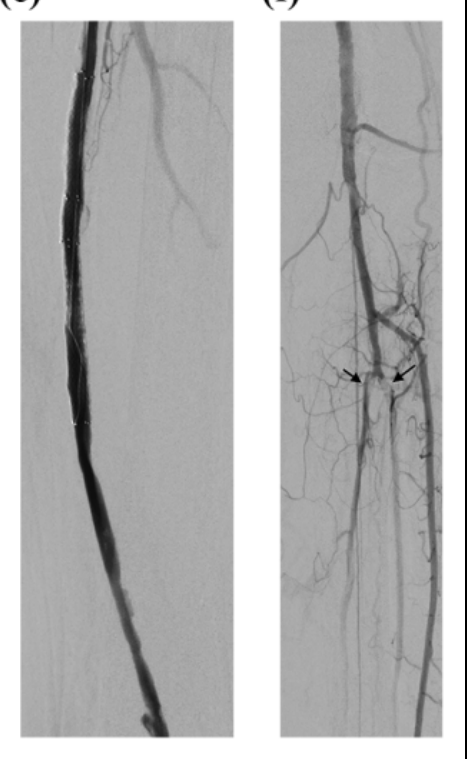

Figure 2. A 72-year-old man suffering from critical limb ischemia with minor tissue loss (Rutherford cat. 5) was admitted for an elective endovascular procedure. a, b, CT VR and axial view show very long and calcified occlusion of the superficial femoral and popliteal artery (TASC D). There is a three-vessel runoff. $c, d$, The angiogram confirms the long occlusion and the multiple collaterals mainly off the profunda femoris artery. e, The occlusion is successfully crossed, and angioplasty performed, but due to flow-limiting dissection in the SFA, two overlapped 7 mm stents $(7 \times 60 \mathrm{~mm}$ and $7 \times 80 \mathrm{~mm}$ Zilver, Cook Medical) are deployed. $\mathrm{f}$, The final angiogram shows DE in the peroneal and posterior tibial artery (arrows), but it was "non-flow-limiting," and therefore, no further intervention was performed. The patient was then discharged without complications. CT indicates computed tomography; SFA, superficial femoral artery; DE, distal embolization.

Table I. Current Evidence on the Different Risk Factors of DE.,

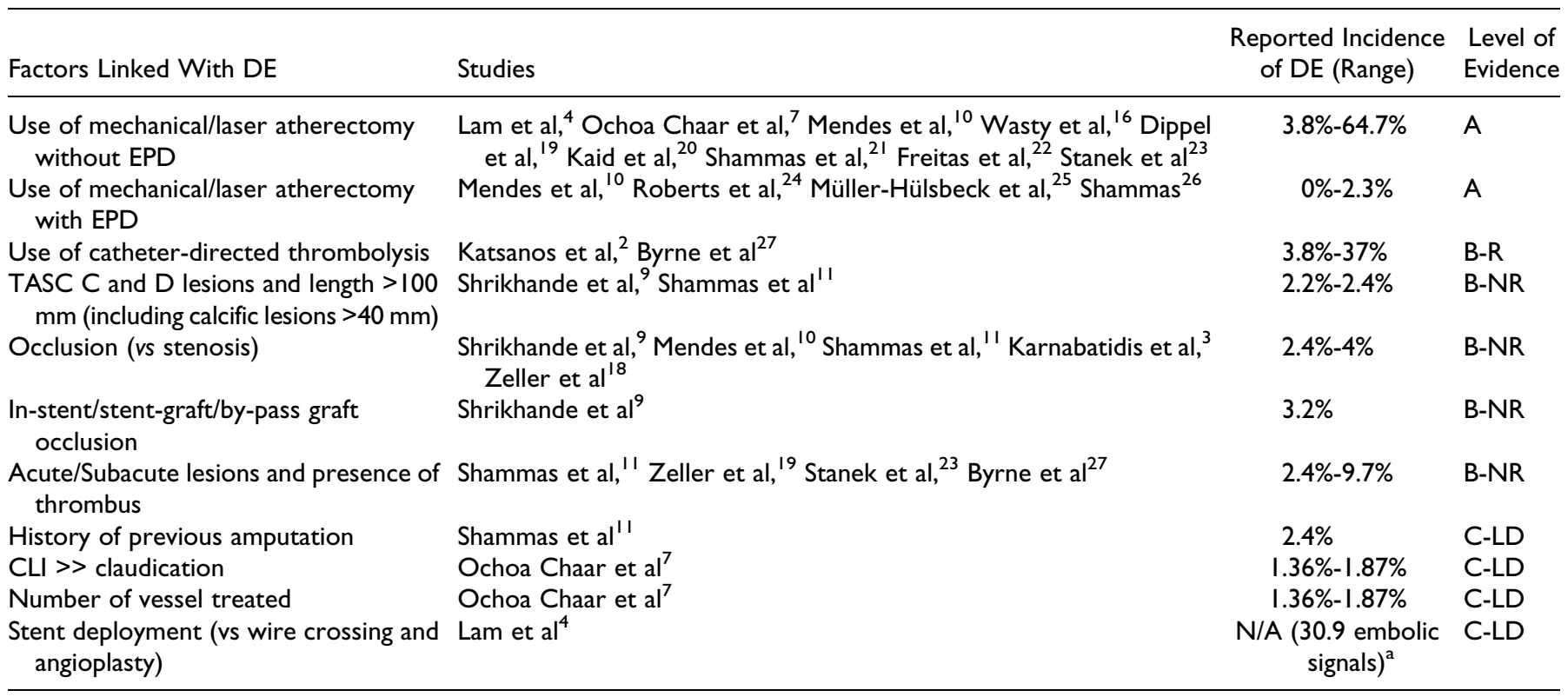

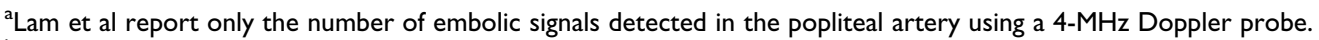

bLevel of evidence according to GRADE system: Level A; level B-R: randomized; level B-NR: non-randomized; level C-LD: limited data. ${ }^{6}$

devices (EPD), especially in the approach to in-stent restenosis. ${ }^{4,9,10,12,18,19}$ More recent studies confirmed those findings: In particular, Shammas et al advocated the use of distal protection devices in long and complex TASC-D occlusions that were found to have a risk of embolism as much as 3.7 times higher than that of TASC A-C lesions. ${ }^{11}$ A history of previous amputation may further increase the likelihood of significant embolization by 4.1 times. ${ }^{11}$ 
Other reported risk factors of $\mathrm{DE}$ are as follows: critical limb ischemia, thrombolysis, in-stent/stent graft stenosis or occlusion, thrombotic lesion, limited distal runoff, previous history of thromboembolic disease, and/or amputation. ${ }^{4,7,9,11}$ The different factors linked with an increased risk of DE are resumed in Table 1.

\section{Prevention strategies}

Prevention strategies aim to reduce the risk of DE during patient preparation and intraoperatively.

\section{Anticoagulation and Antiplatelet Therapy}

Studies have demonstrated that the disruption of plaques after carotid angioplasty may trigger a cascade of events, including the release of plaque debris, deposition of platelets, and formation of thrombus. ${ }^{4}$ This kind of process may also apply to the endovascular treatment of lower limb disease.

For this reason, anticoagulation therapy is indicated with an intravenous dose of unfractioned heparin of 60 to $100 \mathrm{IU}$ per $\mathrm{kg}$, while the intra-arterial heparin administration remains off-label, although no side events have been reported to date. ${ }^{2,32}$ A target activated clotting time between 250 and 300 seconds is advocated. ${ }^{32}$

Although there is fair evidence on the mid- and long-term effects of antiplatelet therapy after treatment, the effect on intraprocedural DE remains uncertain.

Nowadays, the mainstay of all patients with peripheral artery disease is a single antiplatelet agent (aspirin 75-325 $\mathrm{mg}$ or Clopidogrel $75 \mathrm{mg}$ ). In fact, recent studies and guidelines recommend the use of at least one antiplatelet agent that may reduce the platelets reactivity to endovascular maneuvers, hence reducing the risk of clot development. ${ }^{2,3,6}$

Also, dual antiplatelet therapy (aspirin and clopidogrel) may be an option, but it is not specifically recommended for femoropopliteal interventions and the protective effect on vascular events is not well established. ${ }^{2,6,33,34}$

Notably, Spiliopoulos et $\mathrm{al}^{5}$ administered dual antiplatelet therapy in all patients since 5 days before the procedure and they did not detect any macroscopic debris in their cohort, but only microscopic emboli trapped in the distal filter.

However, to define the role of antiplatelet therapy in reducing the risk of DE still requires further large-scale studies and registries.

\section{The EPD}

The clinical use and evidence on EPD are increasing exponentially with the use of mechanical and laser atherectomy devices in complex femoropopliteal lesions. Several types of EPD have been produced, but only two devices are approved by the Food and Drug Administration for collecting debris in the lower limbs. These are the SpiderFX filter, which is to be used only with atherectomy, and the Proteus embolic capture angioplasty balloon catheter, which folds inward after dilation, drawing embolic material inside the formed cavity. Both basket and balloon-based EPD showed high procedure and device success rates $(>95 \%)$, with a limited number of complications. ${ }^{10,12,24,21,25,35,36}$ In the last years, different studies and some trials confirmed a very low rate of DE, making the EPD very effective devices in preventing DE during lower limb interventions and dramatically reducing the risk of DE during mechanical atherectomy. ${ }^{10,12,21,24}$

However, their use requires a certain technical experience as complications have been reported to occur during the procedure, such as dissection or spasm related to device motion, especially in diseased vessels or issues during retrieval.

A recent study ${ }^{35}$ tried to identify the lesions at higher risk of DE (CTO, in-stent restenosis, thrombotic, calcified lesions $>40 \mathrm{~mm}$, and atherosclerotic lesions $>140 \mathrm{~mm}$ ) to be selected for treatment using EPD. However, according to some authors, other important factors such as cost-benefit, operator experience, and other lesion characteristics still deserve further investigation; therefore, additional evidence is still awaited for adequate patients' selection. ${ }^{36}$

Other technical measures. There are general measures that may prevent or reduce the risk of DE, as the regular or continuous flushing of the sheath using heparinized saline solution. Other critical measures are the use of lower profile catheters, careful choice, and manipulation of wires and the minimization of the number of balloon inflations by using long balloons. ${ }^{17}$ In CTO, the subintimal recanalization is deemed by some authors as a lower risk technique for $\mathrm{DE}$, but recent studies performing manual recanalization or recanalization with outback reentry device (Cordis Corporation, Bridgewater, New Jersey) showed DE occurring in $3 \%-5 \%$ of cases. ${ }^{26,37}$

Most recently, Galanakis et $\mathrm{al}^{38}$ showed in 16 patients as direct stenting using bare metal stents may be a valid option in acute short segment lesions, when thrombolysis is contraindicated. They demonstrated no significant residual stenoses in the stented segments, neither DE nor acute in-stent thrombosis. Although this may be considered as a valid option to prevent $\mathrm{DE}$ in acute lesions, the level of evidence is low and further research is warranted.

\section{Management Techniques}

The DE is essentially an iatrogenic hyperacute limb ischemia. As described in several studies, the embolized material can be differentiated between fresh and old thrombus that are associated with calcified debris, cholesterol clefts, and platelets aggregates in different degree according to the age of the stenosis. ${ }^{3,5,20,28}$ Therefore, various composition of the lesion may influence the choice of the different bailout procedures. Indication to treatment of DE may depend on clinical-anatomical characteristics and operator's choice, but generally, it is indicated in flow-limiting DE involving at least one distal artery or a distal branch. ${ }^{11,18}$

In fact, patients experiencing DE require significantly more reinterventions $(20 \%$ vs $3 \%, P<.001)$ and have higher risk for 
major amputations at 30 days compared with patients without DE $(11 \%$ vs $3 \%, P=.02)$ as found by Mendes et al. ${ }^{10}$

In retrospective studies, long-term patency in patients who experienced DE is similar to patients who did not. ${ }^{10,39}$ Target limb primary patency and secondary patency may vary from $60 \%$ to $80 \%$ at 1 year and depend on the type of bailout technique used (ie, AngioJet rheolytic thrombectomy vs standard thrombolysis) and original lesion treated (ie, TASC A vs TASC C/D). ${ }^{10,39}$

Technical and clinical success of salvage procedures are generally high, achieving at least an improvement of the clinical condition in about $80 \%$ of patients experiencing DE. ${ }^{10}$

Over the last decade, the approach to DE has been mainly via classic endovascular techniques (manual suction, mechanical thromboaspiration, thrombolysis), reserving surgery or hybrid approach for the most severe cases (larger or proximal emboli and/or limb at immediate threat) or when endovascular techniques fail. ${ }^{2,7,12}$ More recently, there has been an increasing interest in new endovascular devices/techniques (ie, Angiojet ${ }^{\circledR}$ reolytic thrombectomy-Boston Scientific, Marlborough, Massachusetts, and Penumbra systemPenumbra Inc, Alameda, California) that have the potential to overcome the limits and complications of the classic endovascular techniques or surgery. ${ }^{18,39,40}$

Classic salvage endovascular techniques include suction, mechanical thromboaspiration, and thrombolysis. ${ }^{18}$

Alternative and adjunctive rescue techniques used by some authors $^{10,22,23,27,39,40,41}$ are intra-arterial nitroglycerin injection, AngioJet rheolytic thrombectomy, angioplasty, and stent at the site of the emboli deposit, retrieval of the embolic material using an EPD or over-the-wire embolectomy with Fogarty catheter.

The simplest and fastest approach is mechanical thromboaspiration embolectomy using large lumen guide catheters, with a caliber of 6- to 8-F for the femoral-popliteal segment and 5-F for the below knee arteries.

The catheter is connected to a $60-\mathrm{mL}$ syringe, and the thrombus is forcefully aspirated out of the vessel. This technique is mainly considered as an adjunctive therapy to thrombolysis, reducing the thrombus burden and therefore the need of pharmacological lysis.

Success of thromboaspiration alone has been reported at $31 \%$, but combined with thrombolysis can rise up to $87.3 \%$ in primary acute limb ischemia. ${ }^{7,12}$ Catheter-directed thrombolysis (CDT) is not only the most common and available among the aforementioned techniques, but it is also advantageous as it enables to reach very distal thrombi. Different drugs are usually mentioned, but the most commonly used are the tenecteplase (TNK), recombinant tissue plasminogen activator (rtPA), and urokinase according to different protocols. ${ }^{8,13}$

Moreover, CDT has been combined with mechanical thrombectomy ${ }^{23,27,39,41}$, and with EKOS $^{\circledR}$ (BTG International, Surrey, UK) device for ultrasound-accelerated thrombolysis 42 either for the treatment of iatrogenic DE or for the primary acute and subacute limb ischemia. The AngioJet ${ }^{\circledR}$, a mechanical thromboaspiration device, may be considered the most indicated for hyperacute thrombo-embolism (but not for the atheroembolic loads), ${ }^{28,39}$ showing a high technical success rate when combined with clot trapping using spot stenting and/or intraoperative urokinase thrombolysis (success rate: $92.8 \%^{39}$ in DE). Another recent article found useful selective direct stenting of distal DE in below knee vessels, however, in a limited number of patients. ${ }^{41}$

Also, other devices are theoretically available for the treatment of iatrogenic acute limb ischemia and DE (ie, Penumbra system $)^{18,22,40}$ : A recent single center experience showed high success rates (immediate success rate: $97.7 \%$ ) of the Rotarex debulking device in native acute and subacute femoropopliteal occlusions; nevertheless, further prospective studies are warranted to confirm indications and effectiveness. ${ }^{22}$

The ultrasound-enhanced debulking did not show a clear advantage in technical success over the CDT alone in latest studies. $^{42,43}$

When CDT and mechanical thrombectomy are not indicated or available, an alternative may be the combination of surgical embolectomy with other endovascular techniques, such as Fogarty embolectomy plus angioplasty with or without stenting. These have been used in the treatment of acute limb ischemia, yielding a technical success up to $99.1 \%{ }^{27,44}$

Salvage surgical thrombectomy alone demonstrated high technical success rate in the femoropopliteal segment (above $70 \%$ ), but it is more complex in below knee arteries and generally has shown clear limitations, such as incomplete thrombus clearance (in up to $35 \%$ of cases), residual stenosis/ occlusion due to the presence of underlying chronic atherosclerotic disease (about $28 \%$ of cases), or vessel wall damage (about $2.5 \%$ of cases) ${ }^{44-46}$ In fact, in up to $16 \%$ of cases, CDT may be necessary to complete below knee arterial revascularization, after attempted surgical embolectomy. ${ }^{44,47}$

In addition, morbidity (wound complications, cardiac events, or pulmonary failure/embolism) and mortality (30-day mortality ranges from $13 \%$ to $18 \%$ ) from surgical techniques continue to be high in contemporary practice for patients treated for acute limb ischemia. ${ }^{45-49}$

Davies et $\mathrm{al}^{50}$ showed as only $32 \%$ of patients who had DE, were treated with open embolectomy alone and the remainder with endovascular techniques. Surgical embolectomy (with either femoral or popliteal artery exposure) was mostly performed in the femoropopliteal segment and tibioperoneal trunk; otherwise, endovascular techniques were performed mostly in the tibial arteries and tibioperoneal trunk. The technical success of embolectomy was as high as for endovascular techniques, showing a technical failure rate of $3.2 \%$ and $1 \%$ of patients required a bypass within 90 days. Two-year limb salvage was $68 \pm 8 \%$, and freedom from recurrent symptoms was $69 \pm 3 \%$.

Nevertheless, there remains a lack of data about the role and success of bailout surgery in iatrogenic DE; hence, further research is warranted.

\section{Conclusion}

We have now good evidence (levels A and B) on the physiopathology and risk factors of $\mathrm{DE}$, including lesion 
features on imaging and devices that may permit the best patient/intervention selection, minimizing the risk of DE. Allowing for EPD that showed to lower the risk of DE in endovascular procedures, stronger evidence is awaited on other preventative techniques. Finally, although classic bailout techniques now have a high rate of success limiting worsening ischemia and limb loss, alternative techniques are of interest and further substantial research on these is certainly warranted.

\section{Declaration of Conflicting Interests}

The author(s) declared no potential conflict of interest with respect to the research, authorship, and/or publication of this article.

\section{Funding}

The author(s) received no financial support for the research, authorship, and/or publication of this article.

\section{References}

1. Jaff MR, White CJ, Hiatt WR, et al. An update on methods for revascularization and expansion of the TASC lesion classification to include below-the-knee arteries: a supplement to the intersociety consensus for the management of peripheral arterial disease (TASC II): the TASC steering committee. Ann Vasc Dis. 2015;8(4):343-357.

2. Katsanos K, Tepe G, Tsetis D, Fanelli F. Standards of practice for superficial femoral and popliteal artery angioplasty and stenting. Cardiovasc Intervent Radiol. 2014;37(3):592-603.

3. Karnabatidis D, Katsanos K, Kagadis GC, et al. Distal embolism during percutaneous revascularization of infra-aortic arterial occlusive disease: an underestimated phenomenon. $J$ Endovasc Ther. 2006;13(3):269-280.

4. Lam RC, Shah S, Faries PL, McKinsey JF, Kent KC, Morrissey NJ. Incidence and clinical significance of distal embolization during percutaneous interventions involving the superficial femoral artery. J Vasc Surg. 2007;46(6):1155-1159.

5. Spiliopoulos S, Theodosiadou V, Koukounas V, et al. Distal macro- and microembolization during subintimal recanalization of femoropopliteal chronic total occlusions. J Endovasc Ther. 2014;21(4):474-481.

6. Gerhard-Herman MD, Gornik HL, Barrett C, et al. 2016 AHA/ ACC guideline on the management of patients with lower extremity peripheral artery disease: a report of the American college of cardiology/American Heart Association Task Force on clinical practice guidelines. Circulation. 2017;135(12):e726-e779.

7. Ochoa Chaar CI, Shebl F, Sumpio B, Dardik A, Indes J, Sarac T. Distal embolization during lower extremity endovascular interventions. J Vasc Surg. 2017;66(1):143-150.

8. Patel NH, Krishnamurthy VN, Kim S, et al.; CIRSE and SIR Standards of Practice Committees. Quality improvement guidelines for percutaneous management of acute lower-extremity ischemia. J Vasc Interv Radiol. 2013;24(1):3-15.

9. Shrikhande GV, Khan SZ, Hussain HG, Dayal R, McKinsey JF, Morrissey N. Lesion types and device characteristics that predict distal embolization during percutaneous lower extremity interventions. J Vasc Surg. 2011;53(2):347-352.
10. Mendes BC, Oderich GS, Fleming MD, et al. Clinical significance of embolic events in patients undergoing endovascular femoropopliteal interventions with or without embolic protection devices. J Vasc Surg. 2014;59(2):359-367.

11. Shammas NW, Shammas GA, Dippel EJ, Jerin M, Shammas WJ. Predictors of distal embolization in peripheral percutaneous interventions: a report from a large peripheral vascular registry. $J$ Invasive Cardiol. 2009;21(12):628-631.

12. Shammas NW, Dippel EJ, Coiner D, Shammas GA, Jerin M, Kumar A. Preventing lower extremity distal embolization using embolic filter protection: results of the PROTECT registry. J Endovasc Ther. 2008;15(3):270-276.

13. Karnabatidis D, Spiliopoulos S, Tsetis D, Siablis D. Quality improvement guidelines for percutaneous catheter-directed intra-arterial thrombolysis and mechanical thrombectomy for acute lower-limb ischemia. Cardiovasc Intervent Radiol. 2011; 34(6):1123-1136.

14. Kühn JP, Hoene A, Miertsch M, et al. Intraarterial recombinant tissue plasminogen activator thrombolysis of acute and semiacute lower limb arterial occlusion: quality assurance, complication management, and 12-month follow-up reinterventions. AJR Am J Roentgenol. 2011;196(5):1189-1193.

15. Chin AS, Rubin GD. CT angiography of peripheral arterial occlusive disease. Tech Vasc Interv Radiol. 2006;9(4):143-149.

16. Reekers JA. Regarding "Distal embolism during percutaneous revascularization of infra-aortic arterial occlusive disease: an underestimated phenomenon". J Endovasc Ther. 2006;13(5): 697; author reply 698-699.

17. Razavi MK. Detection and treatment of acute thromboembolic events in the lower extremities. Tech Vasc Interv Radiol. 2011; 14(2):80-85.

18. Zeller T, Schmidt A, Rastan A, et al. New approach to protected percutaneous transluminal angioplasty in the lower limbs. $J$ Endovasc Ther. 2013;20(3):409-419.

19. Dippel EJ, Makam P, Kovach R, et al. Randomized controlled study of excimer laser atherectomy for treatment of femoropopliteal in-stent restenosis: initial results from the EXCITE ISR trial (EXCImer Laser Randomized Controlled Study for Treatment of FemoropopliTEal In-Stent Restenosis). JACC Cardiovasc Interv. 2015;8(1 pt A):92-101.

20. Kaid KA, Gopinathapillai R, Qian F, et al. Analysis of particulate debris after superficial femoral artery atherectomy. $J$ Invasive Cardiol. 2009;21(1):7-10.

21. Shammas NW, Coiner D, Shammas GA, Dippel EJ, Christensen L, Jerin M. Percutaneous lower-extremity arterial interventions with primary balloon angioplasty versus Silverhawk atherectomy and adjunctive balloon angioplasty: randomized trial. J Vasc Interv Radiol. 2011;22(9):1223-1228.

22. Freitas B, Steiner S, Bausback Y, et al. Rotarex mechanical debulking in acute and subacute arterial lesions: single-center experience with 525 patients. Angiology. 2017;68(3):233-241.

23. Stanek F, Ouhrabkova R, Prochazka D. Mechanical thrombectomy using the Rotarex catheter in the treatment of acute and subacute occlusions of peripheral arteries: immedite results, long-term follow-up. Int Angiol. 2013;32(1):52-60. 
24. Roberts D, Niazi K, Miller W, et al. DEFINITIVE $\mathrm{Ca}^{++}$Investigators. Effective endovascular treatment of calcified femoropopliteal disease with directional atherectomy and distal embolic protection: final results of the DEFINITIVE $\mathrm{Ca}++$ trial. Catheter Cardiovasc Interv. 2014;84(2):236-244.

25. Müller-Hülsbeck S, Hümme TH, et al. Final results of the protected superficial femoral artery trial using the FilterWire EZ system. Cardiovasc Intervent Radiol. 2010;33(6):1120-1127.

26. Shammas NW. Subintimal angioplasty and stenting of chronic total femoropopliteal occlusions: is distal protection needed? J Endovasc Ther. 2014;21(4):482-484.

27. Byrne RM, Taha AG, Avgerinos E, Marone LK, Makaroun MS, Chaer RA. Contemporary outcomes of endovascular interventions for acute limb ischemia. J Vasc Surg. 2014;59(4):988-995.

28. Wasty N, Khakwani MZ, Kotev S, et al. Ubiquitous nature of distal athero/thromboembolic events during lower extremity atherectomy procedures involving the superficial femoral artery. Int $J$ Angiol. 2016;25(4):252-257.

29. McKinsey JF, Zeller T, Rocha-Singh KJ, et al; Definitive Le Investigators. Lower extremity revascularization using directional atherectomy: 12-month prospective results of the Definitive Le study. JACC Cardiovasc Interv. 2014;7(8):923-933.

30. Dattilo R, Himmelstein SI, Cuff RF. The compliance $360^{\circ}$ trial: a randomized, prospective, multicenter, pilot study comparing acute and long-term results of orbital atherectomy to balloon angioplasty for calcified femoropopliteal disease. J Invasive Cardiol. 2014;26(8):355-360.

31. Laird JR, Zeller T, Gray BH, et al; LACI Investigators. Limb salvage following laser-assisted angioplasty for critical limb ischemia: results of the LACI multicenter trial. $J$ Endovasc Ther 2006;13(1):1-11.

32. Altenburg A, Haage P. Antiplatelet and anticoagulant drugs in interventional radiology. Cardiovasc Intervent Radiol. 2012; 35(1):30-42.

33. Mendes BC, Oderich GS. Re: distal macro- and microembolization during subintimal recanalization of femoropopliteal chronic total occlusions. J Endovasc Ther. 2015;22(1):146-147.

34. Tepe G, Bantleon R, Brechtel K, et al. Management of peripheral arterial interventions with mono or dual antiplatelet therapy - the MIRROR study: a randomised and double-blinded clinical trial. Eur Radiol. 2012;22(9):1998-2006.

35. Krishnan P, Tarricone A, Purushothaman KR, et al. An algorithm for the use of embolic protection during atherectomy for femoral popliteal lesions. JACC Cardiovasc Interv. 2017;10(4):403-410.

36. Armstrong EJ, Waldo SW. Prevention of distal embolization during peripheral vascular interventions: filtering the evidence. JACC Cardiovasc Interv. 2017;10(4):411-412.

37. Kitrou P, Parthipun A, Diamantopoulos A, Paraskevopoulos I, Karunanithy N, Katsanos K. Targeted true lumen re-entry with the outback catheter: accuracy, success, and complications in 100 peripheral chronic total occlusions and systematic review of the literature. J Endovasc Ther. 2015;22(4):538-545.
38. Galanakis N, Kontopodis N, Peteinarakis I, Kehagias E, Ioannou $\mathrm{CV}$, Tsetis D. Direct stenting in patients with acute lower limb arterial occlusions: immediate and long-term results. Cardiovasc Intervent Radiol. 2017;40(2):192-201.

39. Spiliopoulos S, Katsanos K, Fragkos G, Karnabatidis D, Siablis D. Treatment of infrainguinal thromboembolic complications during peripheral endovascular procedures with AngioJet rheolytic thrombectomy, intraoperative thrombolysis, and selective stenting. J Vasc Surg. 2012;56(5):1308-1316.

40. Gandini R, Merolla S, Chegai F, Del Giudice C, Stefanini M, Pampana E. Foot embolization during limb salvage procedures in critical limb ischemia patients successfully managed with mechanical thromboaspiration: a technical note. J Endovasc Ther. 2015;22(4):558-563.

41. Krokidis M, Ali T, Hilliard N, et al. Intraprocedural distal embolization after femoropopliteal angioplasty: is there a role for below-the-knee stents? Cardiovasc Intervent Radiol. 2017; 40(8):1155-1163.

42. Schrijver AM, van Leersum M, Fioole B, et al. Dutch randomized trial comparing standard catheter-directed thrombolysis and ultrasound-accelerated thrombolysis for arterial thromboembolic infrainguinal disease (DUET). J Endovasc Ther. 2015;22(1): 87-95.

43. Schernthaner MB, Samuels S, Biegler P, Benenati JF, Uthoff H. Ultrasound-accelerated versus standard catheter-directed thrombolysis in 102 patients with acute and subacute limb ischemia. J Vasc Interv Radiol. 2014;25(8):1149-1156.

44. de Donato G, Setacci F, Sirignano P, Galzerano G, Massaroni R, Setacci $\mathrm{C}$. The combination of surgical embolectomy and endovascular techniques may improve outcomes of patients with acute lower limb ischemia. J Vasc Surg. 2014;59(3):729-736.

45. Branco BC, Montero-Baker MF, Mills JL Sr. The pros and cons of endovascular and open surgical treatments for patients with acute limb ischemia. J Cardiovasc Surg (Torino). 2015;56(3):401-407.

46. O'Connell JB, Quiñones-Baldrich WJ. Proper evaluation and management of acute embolic versus thrombotic limb ischemia. Semin Vasc Surg. 2009;22(1):10-16.

47. Kempe K, Starr B, Stafford JM, et al. Results of surgical management of acute thromboembolic lower extremity ischemia. J Vasc Surg. 2014;60(3):702-707.

48. Casillas-Berumen S, Sadri L, Farber A, et al. Morbidity and mortality after emergency lower extremity embolectomy. J Vasc Surg. 2017;65(3):754-759.

49. Taha AG, Byrne RM, Avgerinos ED, Marone LK, Makaroun MS, Chaer RA. Comparative effectiveness of endovascular versus surgical revascularization for acute lower extremity ischemia. J Vasc Surg. 2015;61(1):147-154.

50. Davies MG, Bismuth J, Saad WE, et al. Implications of in situ thrombosis and distal embolization during superficial femoral artery endoluminal intervention. Ann Vasc Surg. 2010;24(1): 14-22. 